\title{
Composizione in antociani e flavonoli di vini prodotti nel territorio svizzero
}

\author{
D. Marchi, D. Lanati, G. Mazza e P. Cascio
}

Enosis s.r.1., 11043, Fubine (AL), Italia

\begin{abstract}
Sintesi. In questa nota sono presentati i risultati delle determinazioni del profilo degli antociani e dei flavonoli di vini prodotti con uve di alcune varietà a frutto colorato prodotte a Nord della Svizzera, nel cantone di Schaffhausen, nella regione di Klettgau. I vini sono stati ottenuti da uve delle varietà di Vitis vinifera Cabernet Dorsa, Gamaret, Dornfelder, Acolon e di un incrocio interspecifico Sylvaner $\times$ Müller Thurgau $\times$ Chambourcin (Regent). Il profilo degli antociani dei vini delle varietà di Vitis vinifera studiate è risultato a netta prevalenza di molecole trisostituite all'anello laterale (soprattutto malvidina-3-glucoside e suoi derivati acilati). Il vino Regent si è rivelato ricco di antociani 3,5-diglucosidi (soprattutto della malvidina) che hanno superato in proporzione i monoglucosidi. Di rilevante interesse è risultato il profilo dei flavonoli per la presenza nei vini, appena dopo la fine della fermentazione alcolica, di quercetina aglicone, di solito assente nell'uva. I vini Acolon e Regent sono risultati i più ricchi di quercetina aglicone. L'origine della quercetina aglicone nei vini esaminati in questo lavoro è stata attribuita all'idrolisi che le forme glicosilate di questo flavonolo hanno subito, probabilmente per via enzimatica, già durante la macerazione fermentativa.
\end{abstract}

\section{Introduzione}

Negli organi fotosintetici, di trasporto e nei frutti colorati di Vitis vinifera sono presenti cinque antocianine (monoglucosidi, -3-G) e le loro forme acilate nella posizione 6 del glucosio. Anche una sesta antocianina, la Pelargonidina-3-G è stata segnala, ma la sua importanza quantitativa è risultata solo marginale (He et al., 2012) [1]. Le percentuali delle singole antocianine e dei loro derivati acilati (acetati, p-cumarati e caffeati) presenti nelle bucce dell'uva rappresentano una sorta di "fingerprint" varietale in quanto nella biosintesi di questi composti la componente genetica varietale ha una influenza molto maggiore di quelle climatiche, ambientali e colturali. Queste ultime interessano maggiormente le cultivar a prevalenza di molecole disostituite all'anello laterale (B) (Cianidina3-G e Peonidina-3-G) (Squadrito et al., 2010) [2]. Le differenze varietali più evidenti riguardano i) le percentuali delle antocianine non acilate (le percentuali delle acilate e delle non acilate sono simili), ii) il rapporto antocianine acilate/antocianine non acilate, iii) il rapporto derivati acetati/derivati p-cumarati, iv) la presenza di derivati acilati. Nelle cultivar diverse dalla Vitis vinifera e negli ibridi colorati possono essere presenti derivati diglucosilati (-3,5-DG) nelle posizioni 3 (anello C) e 5 (anello A). Nel passaggio da uva a vino il profilo degli antociani dell'uva si mantiene sufficientemente invariato nelle cultivar a prevalenza di antociani trisostituiti nell'anello laterale (Delfinidina-3G, Petunidina-3-G e Malvidina-3$\mathrm{G})$, mentre può subire rilevanti cambiamenti nelle cultivar a prevalenza di disostituiti (Squadrito et al., 2010) [2]. Le ragioni delle differenze uva-vino sono da ricercare: a) in fase prefermentativa i) nella diversa velocità di diffusione delle singole antocianine dalle bucce nel mosto e ii) nel coinvolgimento delle antocianine nelle reazioni di ossidazione catalizzate dalle Polifenol Ossidasi dell'uva (PPO), b) in fase fermentativa: i) nel diverso assorbimento delle singole antocianine da parte dei lieviti, ii) nel loro diverso comportamento nei riguardi delle reazioni idrolitiche via carbinolo-calcone (Guerra, 1997) [3] e iii) nell'inizio delle reazioni di polimerizzazione con $\mathrm{i}$ tannini, c) in fase postfermentativa soprattutto in queste due ultime reazioni ii)b e iii)b e nelle reazioni di ossidazione. Per maggiori informazioni sulle differenze fra i profili degli antociani dell'uva e del vino si rimanda, comunque, al lavoro di Squadrito et al. (2010) [2]. Scarse notizie riguardano l'evoluzione del profilo degli antociani dall'uva al vino per le cultivar in cui sono presenti antociani diglucosidi in quanto gli studi fino ad ora effettuati hanno riguardato soprattutto la Vitisvinifera. Inoltre, mentre il profilo dei flavonoli delle cultivar di Vitis vinifera è stato studiato per l'importanza di questi composti nei riguardi degli aspetti salutistici del consumo del vino e per l'intorbidamento che può causare l'insolubilizzazione della Quercetina aglicone (Lanati et al., 2014) [4], il profilo dei flavonoli delle cultivar diverse dalla Vitis vinifera e la sua evoluzione nel passaggio da uva a vino e durante la conservazione del vino rimangono sostanzialmente da determinare.

Allo scopo di ottenere informazioni sulle influenze tecnologiche ed ambientali sul profilo degli antociani e dei flavonoli, in questo lavoro sono stati studiati e confrontati con quelli delle rispettive uve (quando disponibili) i profili dei composti in questione di vini di cinque cultivar di Vitis vinifera (Cabernet Dorsa, Dornfelder, Gamaret, Acolon e Dakapo)) e di un ibrido interspecifico (Regent) prodotti in 
Table 1. Composizione dei vini Cabernet Dorsa, Acolon, Dornfelder, Dakapo, Gamaret, e Regent. *media di due vini diversi.

\begin{tabular}{|lccccccccc|} 
& $\begin{array}{c}\text { Cabernet } \\
\text { Dorsa }\end{array}$ & Acolon & $\begin{array}{c}\text { Dornfelder } \\
\mathbf{1}\end{array}$ & $\begin{array}{c}\text { Dornfelder } \\
\mathbf{2}\end{array}$ & Dakapo* $^{*}$ & Gamaret & $\begin{array}{c}\text { Regent } \\
\text { Rosso }\end{array}$ & $\begin{array}{c}\text { Regent } \\
\text { Rosé }\end{array}$ \\
\hline G.A.\% vol & 13,81 & 13,01 & 11,05 & 12,17 & 11,45 & 13,78 & 14,15 & 14,89 \\
\hline Ac. titolab. g/L & 7,1 & 7,7 & 5,7 & 7,1 & 7,45 & 5,9 & 7,3 & 5,4 \\
\hline Ac. vol.g/L & 0,23 & 0,26 & $<0,1$ & 0,15 & 0,16 & 0,33 & 0,16 & 0,42 \\
\hline pH & 3,51 & 3,4 & 3,60 & 3,48 & 3,51 & 3,54 & 3,54 & 3,53 \\
\hline Ac. Mal.g/L & 1,92 & 2,44 & 1,85 & 1,87 & 2,38 & 0,73 & 2,61 & 2,23 \\
\hline Ac. Latt.g/L & 0,81 & 0,71 & 1,1 & 0,87 & 1,14 & 0,77 & 0,71 & 0,36 \\
\hline Antoc. tot.mg/L & 1237 & 1280 & 923 & 1245 & 1869 & 1064 & 1701 & 616 \\
\hline Flavonoidi tot.mg/L & 2009 & 2360 & 1490 & 2144 & $>3000$ & $>3000$ & 2995 & 1048 \\
\hline
\end{tabular}

Svizzera nella vendemmia 2018. Tale studio si inquadra in un progetto più generale riguardante la conoscenza della composizione fenolica dei vitigni coltivati nelle più rinomate zone vitivinicole dell'Europa e dell'Asia e l'ottimizzazione dell'aspetto varietale delle tecniche di vinificazione.

\section{Materiali e metodi}

I vini sono stati prelevati alla fine della fermentazione alcolica (ottobre 2018) da una cantina della regione Klettgau, vicino alla città di Hallau Svizzera. Essi sono stati prodotti con tecniche classiche, escluso i vini Dakapo e Regent i cui mosti sono stati ottenuti per termovinificazione. Il grado alcolico, l'acidità titolabile, l'acidità volatile, il $\mathrm{pH}$, sono stati determinati con il FossWineScann, gli acidi malico e lattico per via enzimatica, gli antociani totali e i flavonoidi totali per spettrofotometria (Corona et al., 2015) [5], il profilo degli antociani per HPLC col metodo OIV-MA-AS315-11 per iniezione diretta del vino, e il profilo dei flavonoli per HPLC dopo preconcentrazione del campione $(10 \mathrm{~mL}$ di vino sono stati estratti con etil acetato; dopo evaporazione del solvente sotto vuoto il residuo è stato ripreso con $2 \mathrm{~mL}$ della miscela $\mathrm{H}_{3} \mathrm{PO}_{4} 10^{-3} \mathrm{M}$ in acqua: metanolo 60:40)

Condizioni cromatografiche per l'analisi dei Flavonoli per HPLC:

HPLC-DAD Perkin-Elmer mod. Series 200 solvente A: $\mathrm{H}_{3} \mathrm{PO}_{4} 10^{-3} \mathrm{M}$ in acqua; solvente $\mathrm{B}$ : metanolo LiChrosolv Merck; da $0 \%$ a $5 \%$ di B in 5 min., a $10 \%$ di B in 5 min., a $30 \%$ di B in 15 min., a $60 \%$ di B in 10 min., a $100 \%$ di $\mathrm{B}$ in $10 \mathrm{~min} ., 100 \%$ di $\mathrm{B}$ per $10 \mathrm{~min}$., a $5 \%$ di B in $5 \mathrm{~min}$. Flow: $0,8 \mathrm{~mL} / \mathrm{min}$., $\lambda: 360 \mathrm{~nm}$, volume iniettato: $20 \mu \mathrm{L}$,

Colonna:LiChrospher ${ }^{\circledR} 100$ RP-18 $(5 \mu \mathrm{m})-$ LiChroCart $^{\circledR}$ 250-4, Merck

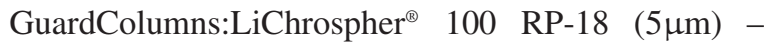
LiChroCart ${ }^{\circledR}$ 4-4, Merck.

\section{Risultati e discussione}

I vini Cabernet Dorsa, Acolon, Gamaret e, soprattutto Regent esaminati in questo lavoro hanno presentato un grado alcolico \% vol (Table 1) piuttosto elevato per la zona del centro Europa in cui sono stati prodotti. Questo implica che, a causa del cambiamento climatico, è attualmente possibile ottenere, anche in zone diverse da quelle mediterranee o calde in genere, vini ricchi di alcol. Tale composizione è risultata bilanciata da acidità titolabili piuttosto elevate e da $\mathrm{pH}$ medio bassi (esclusi Dornfelder 1 e Regent Rosé), in linea con la zona di origine. È prevedibile, comunque, un aumento del $\mathrm{pH}$ dopo fermentazione malolattica. Un altro effetto del cambiamento climatico è rappresentato dal contenuto non elevato di acido malico dei vini sopra menzionati. Il basso grado alcolico dei vini Dornfelder 1 e Dakapo, invece, potrebbe indicare che le uve di queste cultivar siano state raccolte precocemente. Il pH elevato del Dorfelder 1 e lo scarso tenore di acido malico, tuttavia, suggeriscono che la capacità di sintesi degli zuccheri di queste ultime cultivar non è stata elevata nell'ambiente considerato. Tutti i vini esaminati, inoltre si sono rivelati ricchi di antociani e di flavonoidi, probabilmente adatti a sostenere un periodo anche lungo di affinamento, almeno quelli dotati di un contenuto in alcol più elevato.

La composizione percentuale degli antociani dei vini elaborati nel territorio svizzero è stata confrontata con quella delle uve riportata nella letteratura enologica internazionale. La Fig. 1 mostra che il profilo degli antociani del vino Cabernet Dorsa (incrocio Dornfelder $\times$ Cabernet Sauvignon, come dichiarato dal costitutore, in realtà incrocio Blaufränkisch $\times$ Dornfelder) differisce dai profili delle uve Cabernet Sauvignon T (Mattivi et al., 2006) [6] e Cabernet Sauvignon Sicilia (nostre esperienze) soprattutto per il rapporto acetati/cinnamati (somma di pcumarati e caffeati) che nei Cabernet sauvignon è apparso sensibilmente maggiore di 1 . Le differenze sono risultate ancora più rilevanti nei riguardi delle uve Blaufränkisch (Balík et al., 2013) [7], una cultivar a netta prevalenza dei derivati cinnamati sugli acetati che complessivamente ricorda i profili delle cultivar mediterranee. Il profilo degli antociani del vino Cabernet Dorsa è sembrato più simile a quello dell'uva e del vino Dornfelder che rappresenta uno dei suoi genitori. Le differenze fra i profili degli antociani del vino Cabernet Dorsa e dell'uva Dornfelder (Balík et al., 2013) [7], hanno riguardato soprattutto le percentuali più basse di Cianidina-3G e di Peonidina-3G del Cabernet Dorsa, in linea con quanto atteso in quanto, come riportato nell'introduzione, questi due antociani sono coinvolti in fase prefermentativa nelle reazioni di ossidazione indotte dalle PPO dell'uva. Le percentuali più elevate dei derivati acetati e cinnamati del vino Cabernet Dorsa rispetto al vino Dornfelder, suggeriscono che anche l'uva Cabernet Dorsa possedeva questo carattere, forse con un rapporto acetati/cinnamati appena minore di 1 . Quest'ultima deduzione si accorda col fatto che, come atteso dal profilo dell'uva, nei vini Dornfelder il rapporto 


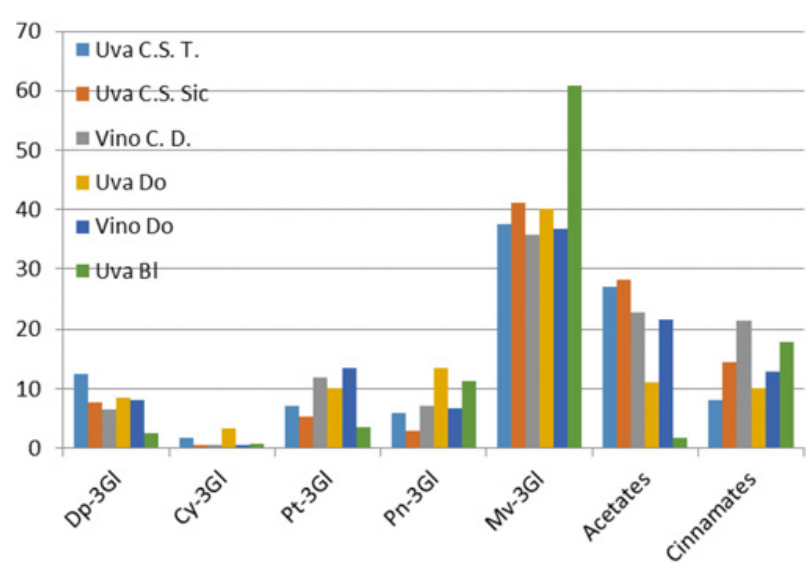

Figure 1. Confronto fra i profili degli antociani di uve, Cabernet Sauvignon (Mattivi et al., 2006), Cabernet Sauvignon Sicilia, Vino Cabernet Dorsa (Svizzera), uva Dornfelder (BalíK et al., 2013, vino Dornfelder (Svizzera), uva Blaufränkisch (BalíK et al., 2013).

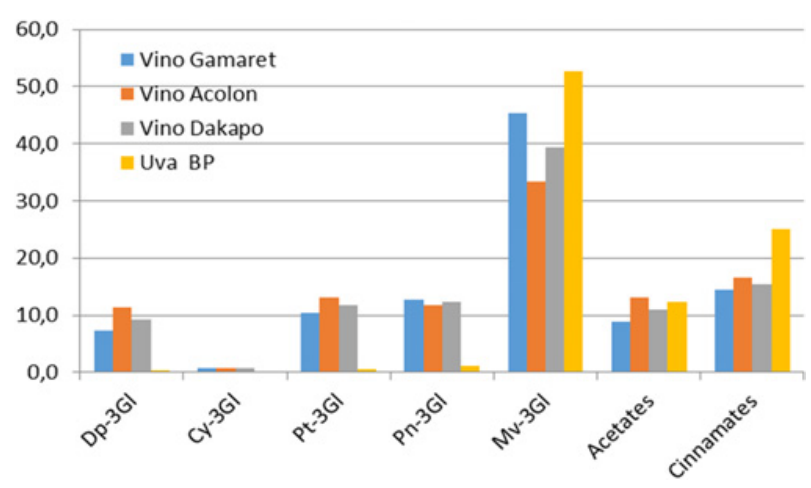

Figure 2. Confronto fra i profili degli antociani di vini Gamaret, Acolon, Dakapo (Svizzera) e uva BlauerPortugieser (BalíK et al., 2013).

acetati/cinnamati è risultato sensibilmente maggiore di 1 mentre nell'uva, solo appena maggiore di 1 . I profili degli antociani non acilati dei vini Cabernet Dorsa e Dornfelder sono risultati, invece, sovrapponibili.

Le differenze fra le due uve Cabernet Sauvignon hanno riguardato sia le percentuali degli antociani non acilati diversi dalla Malvidina-3-G, sia, soprattutto, il rapporto acetati/cinnamati che nel Cabernet Sauvignon $\mathrm{T}$ è apparso più elevato del Cabernet Sauvignon Sicilia. Entrambe queste differenze possono essere attribuite a fattori climatici (intensità del contatto con la radiazione solare, temperatura). Questo risultato, tuttavia, non si accorda col fatto che la maggior insolazione a cui probabilmente sono state sottoposte le uve della Sicilia avrebbe dovuto determinare un aumento della percentuale degli acetati (Haselgrove et al., 2000 [8]; nostre esperienze non pubblicate) e, di conseguenza, un rapporto acetati/cinnamati più elevato nel Cabernet Sauvignon Sicilia.

I profili degli antociani dei vini Gamaret (incrocio Gamay $\times$ Reichensteiner), Acolon (incrocio Blaufränkisch $\times$ Dornfelder, fratello del Cabernet Dorsa) e Dakapo (incrocio Deckrot $\times$ BlauerPortugieser, indicato come teinturier, come il suo genitore Deckrot) sono risultati molto simili (Fig. 2). Nelle uve di questi vitigni, rispetto ai rispettivi vini, potrebbero essere state più elevate

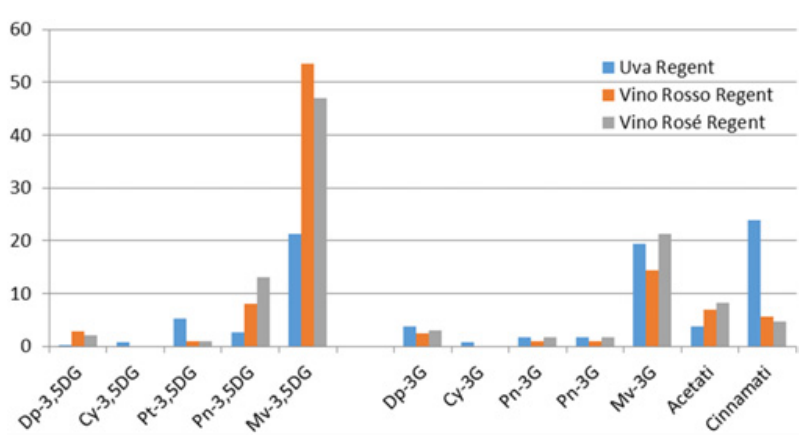

Figure 3. Confronto fra i profili degli antociani di uva Regent (BalíK et al., 2013) e vini Regent (Svizzera).

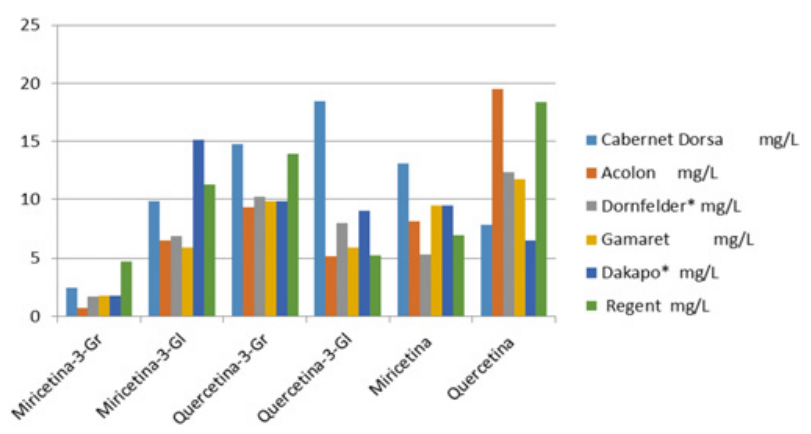

Figure 4. Flavonoli di vini della Svizzera 2018, mg/L. Gr: glucuronide; Gl: Glucoside; * media di due vini diversi.

le percentuali della Delfinidina-3-G, della Cianidina-3-G, della Peonidina-3-G e dei derivati cinnamati in quanto, di solito, le percentuali di questi composti diminuiscono nel passaggio da uva a vino, a favore della Malvidina3-G e dei derivati acetati. A queste trasformazioni e all'effetto della termovinificazione, probabilmente, si deve il profilo a prevalenza di antociani trisostituiti del vino Dakapo che non sembrerebbe accordarsi con la natura di teinturier di questa cultivar. Nella polpa delle cultivar teinturier, infatti, prevalgono gli antociani disostituiti che nel vino in questione sono molto meno rappresentati dei trisostituiti. Non è possibile, comunque, un confronto fra i profili degli antociani dell'uva Dakapo e dei suoi genitori in quanto non abbiamo trovato in letteratura né il profilo degli antociani di questa stessa cultivar, né dell'uva Deckrot (le somiglianze con la BlauerPortugieser riguardano soprattutto le percentuali dei derivati acilati, tenendo conto delle trasformazioni indotte dal processo di macerazione fermentativa). Sulla base di quanto noto dalla letteratura e da quanto riportato da Balík et al., (2013) [7], si può ipotizzare che nell' Acolon è prevalsa la componente Dornfelder sulla Blaufränkisch. Il profilo degli antociani del Gamaret (Gamay $\times$ Reichensteiner) differisce da quello del Gamay in cui le molecole disostituite costituiscono una frazione importante del totale. Reichensteiner è una cultivar a frutto bianco. Questo risultato implica che il profilo degli antociani degli incroci possa essere diverso da quello dei genitori. I vitigni esaminati, comunque, sono ricchi di antociani (Table 1) fra i quali prevalgono quelli della classe dei trisostituiti.

L'uva Regent (Sylvaner $\times$ Muller Thurgau $\times$ ibrido interspecifico Chambourcin) contiene antociani 3,5diglucosidi (soprattutto malvidina-3,5-DG) (Fig. 3). Fra gli antociani monoglucosidi sono prevalsi nettamente 
Table 2. Flavonoli di vini della Svizzera 2018, mg/L. Gr: glucuronide; Gl: Glucoside; * media di due vini diversi.

\begin{tabular}{lcccccc} 
& $\begin{array}{c}\text { Cabernet } \\
\text { Dorsa }\end{array}$ & Acolon & Dornfelder* & Gamaret & Dakapo* & Regent \\
\hline Miricetina-3-Gr & 2,46 & 0,73 & 1,70 & 1,74 & 1,80 & 4,72 \\
\hline Miricetina-3-Gl & 9,82 & 6,55 & 6,93 & 5,94 & 15,10 & 11,25 \\
\hline Quercetina-3-Gr & $\mathbf{1 4 , 7 2}$ & $\mathbf{9 , 2 8}$ & $\mathbf{1 0 , 2 5}$ & $\mathbf{9 , 8 7}$ & $\mathbf{9 , 8 4}$ & $\mathbf{1 3 , 9 2}$ \\
\hline Quercetina-3-Gl & $\mathbf{1 8 , 4 6}$ & $\mathbf{5 , 1 6}$ & $\mathbf{7 , 9 5}$ & $\mathbf{5 , 8 8}$ & $\mathbf{8 , 9 8}$ & $\mathbf{5 , 2 1}$ \\
\hline Miricetina & 13,08 & 8,07 & 5,31 & 9,45 & 9,48 & 6,98 \\
\hline Quercetina & $\mathbf{7 , 8 2}$ & $\mathbf{1 9 , 4 9}$ & $\mathbf{1 2 , 3 2}$ & $\mathbf{1 1 , 7 0}$ & $\mathbf{6 , 5 4}$ & $\mathbf{1 8 , 3 8}$ \\
\hline
\end{tabular}

la Malvidina-3-G e i suoi derivati cinnamati (Balík et al., 2013) [7]. Inoltre il rapporto derivati acetati/derivati cinnamati è risultato sensibilmente minore di 1 . Per effetto della fermentazione e, probabilmente anche della termovinificazione, è stato osservato in entrambi i vini Regent un rilevante incremento delle percentuali degli antociani diglucosidi mentre le percentuali dei derivati acilati sono diminuite e il rapporto acetati/cinnamati è diventato maggiore di 1 . L'incremento delle percentuali dei diglucosidi potrebbe essere dovuto alla maggiore velocità di diffusione dalle bucce nel mosto di queste molecole in fase prefermentativa e alla loro maggiore stabilità in soluzione. La diminuzione delle percentuali dei cinnamati suggerisce, invece, che la termovinificazione oltre a favorire il loro passaggio dalle bucce nel mosto, ne ha causato la degradazione idrolitica. Considerate le scarse notizie che riguardano l'evoluzione del profilo degli antociani delle cultivar che contengono diglucosidi, nel passaggio da uva a vino, le considerazioni sopra svolte dovranno essere supportate da ulteriori ricerche.

I dati riportati in Table 2 mostrano che i contenuti in flavonoli (glicosidi e agliconi della Quercetina e della Miricetina) hanno subito una evoluzione diversa in ciascuno dei vini esaminati. Nei vini Acolon e Regent e un po' meno in Gamaret e Dornfelder è stata osservata una sensibile idrolisi dei glicosidi della Quercetina (soprattutto della forma glucoside), come è possibile dedurre dal confronto fra i contenuti delle forme glicosilate e aglicone di questo flavonolo. Tale idrolisi sembra essere stata meno rilevante in Dakapo, e soprattutto in Cabernet Dorsa, in cui il contenuto della Quercetina aglicone è risultato minore della somma dei contenuti delle forme glicosilate. L'idrolisi delle forme glicosilate della Miricetina ha seguito una via diversa, come dimostrano i dati della Table 2. Infatti, è stato osservato un rapporto Miricetinaaglicone/glicosidi della Miricetina maggiore di 1 in Cabernet Dorsa, Acolon e Gamaret, minore di 1 in Dornfelder, Dakapo e Regent, mentre il rapporto Quercetina aglicone/ glicosidi della Quercetina è risultato maggiore di 1 in Acolon, circa 1 in Regent e minore di 1 in Cabernet Dorsa, Dornfelder, Gamaret e Dakapo. I dati riportati in Table 2 mostrano anche che l'idrolisi della Quercetina-3-glucoside (-3-Gl) è stata più sensibile di quella della Quercetina-3-glucuronide (-3-Gr) (questa conclusione è supportata da nostre ricerche non pubblicate). Inoltre la Quercetina-3-Gl ha subito una consistente idrolisi soprattutto nei vini Acolon, Regent, Dornfelder e Gamaret. Trattandosi di vini del 2018, dello stesso territorio, questi risultati suggeriscono che l'idrolisi della Quercetina-3-Gl e, sebbene in minor misura, della Quercetina-3-Gr, sia avvenuta già nella fase fermentativa, probabilmente per via enzimatica (le differenze fra i vini possono essere attribuite a differenze varietali). In fase di maturazione e affinamento del vino l'idrolisi della Quercetina avviene, invece, per via chimica, catalizzatore $\mathrm{H}^{+}$. Alla Quercetina liberata per quest'ultima via si devono i precipitati osservati in numero sempre più elevato nei vini di certi territori vitivinicoli (Lanati et al., 2014) [4]. I contenuti di quercetina aglicone dei vini Acolon e Regent, sensibilmente maggiori di $10 \mathrm{mg} / \mathrm{L}$ (limite di guardia da noi proposto sulla base di un numero sufficientemente elevato di osservazioni), potrebbero indurre la formazione di un precipitato di questo composto in bottiglia (solubilità della Quercetina circa $5 \mathrm{mg} / \mathrm{L}$, Boulton, 1997) [9]. Trattandosi di vini dal colore intenso (alto contenuto in antociani), sembra verosimile che la quercetina aglicone sia stabilizzata in soluzione per il suo coinvolgimento in complessi di copigmentazione, malgrado il suo contenuto sia superiore alla sua solubilità. Se non è coinvolta nelle reazioni di ossidazione durante la fase di maturazione del vino, la quercetina si può insolubilizzare dopo l'imbottigliamento producendo un precipitato voluminoso. Queste osservazioni, tuttavia, richiedono opportune verifiche, considerata la scarsità delle conoscenze e, in pratica, la mancanza di ricerche in questo campo. I contenuti elevati di derivati della quercetina osservati nei vini in questo lavoro suggeriscono una influenza del cambiamento climatico e delle pratiche colturali sulla sintesi dei flavonoli. A causa della maggiore insolazione e dell'assottigliamento dello strato di ozono in tutte le zone vitivinicole, la comune pratica della defogliazione per esporre i grappoli alla luce del sole in fase di maturazione, porta sicuramente ad una maggiore sintesi di flavonoli che va tenuta sotto controllo per i problemi di stabilità del vino sopra menzionati. Infine, il fatto che il Regent si sia rivelato una cultivar ricca di flavonoli, indica che è necessario un approfondimento dello studio di questi composti sia nelle cultivar non Vitis vinifera, sia nei territori in cui il cambiamento climatico ha indotto un incremento del contatto della radiazione solare con i grappoli dell'uva.

\section{Letteratura citata}

[1] F. He, N.-N. Liang, L. Mu, Q.-H. Pan, J. Wang, M.J. Reeves, C.-Q. Duan, Molecules 17, 1571 (2012)

[2] M. Squadrito et al. J. Int. Sci. VigneVin 44, 167 (2010) 
[3] C.C. Guerra Recherchessur les interactions anthocyanes-flavanols: Application à l'interpretation chimique de la couleur des vins rouges (Thèse de Doctorat à l'Université de Bordeaux, 1997), p. 2

[4] D. Lanati, D. Marchi, P. Cascio. Precipitati di quercetina nei vini. $37^{\circ}$ Congresso OIV (2014)

[5] C. Onofrio, M. Squadrito, G. Vento, A. Tirelli, Rocco Di Stefano Over-evaluation of totalflavonoids in grape skinextracts containing sulphur dioxide Food Chemistry 172, 537 (2015)
[6] F. Mattivi, R.Guzzon, U. Vrhovsek, M. Stefanini, R. J. Agric. Food Chem. 54, 7692 (2006)

[7] J. Balik, M. Kumšta, R. Otakar Comparison of anthocyanins present in grapes of Vitisvinifera L. varieties and interspecific hybrids grown in the Czech Republic Chemical Papers 671285 (2013)

[8] L. Haselgrove, D. Botting, R. van Heeswijck, P.B. Høi, P-R. Dry, C. Ford, P.G. Iland. Aust. J. Grape Wine Res. 6, $141(2000)$

[9] R. Boulton. Am. J. Enol. Vitic. 52, 67 (2001) 